\title{
Identification of Damage on a Substructure with Measured Frequency Response Functions
}

\author{
Nam-Gyu Park \\ Nuclear Fuel Design Department. Korea Nuclear Fuel Co. LTD., \\ 493, Deogjin-dong. Youseong-gu, Daejeon 305-353, Korea \\ Youn-sik Park* \\ Center for Noise and Vibration Control, Department of Mechanical Engineering \\ Korea Advanced Institute of Science and Technology, \\ Science Town, Daejeon 305-70I, Korea
}

\begin{abstract}
Recently the authors tried to find damage position only using measured frequency response functions. According to their work, it seems that the algorithm is very practical since it needs only measured frequency responses while other methods require exact analytic model. But when applying the method to a real structure, it requires lots of experiment. The authors, in this time, propose a method to reduce its experimental load by detecting damage within a substructure. This method searches damages not within an entire structure but within substructures. In addition, damage severity was treated in this paper since it is worthy to know damage severity. Optimization technique is used to estimate damage level using measured responses and damage model. Two test examples, a plate and a jointed structure, are chosen to verify the suggesting method.
\end{abstract}

Key Words : Damage Identification, Frequency Response Functions

\section{Introduction}

Damage such as a crack in a structure makes it vulnerable to external load thus it is necessary to find it before the structure goes to failure. Recently a kind of non-destructive method based on vibration signal is getting more attention to detect damage within a structure (Doebling et al., 1997; Marwala and Heyns, 1998 ; Choi and Lee, 2003).

The reason for people paying attention to this vibration signal analysis method is that many related fields to structural dynamics such as structure modification are already well developed

\footnotetext{
- Correspondirg Author,

E-mail : yspark@kaist.ac.kr

TEL : +82-42-869-3020; FAX : +82-42-869-8220

Center for Noise and Vibration Control, Department of Mech. Engineering Korea Advanced Institute of Science and Technology, Science Town, Daejeon 305-701, Korea. (Manuscript Received March 21, 2005; Revised July 28, 2005)
}

and vibration measurement is not difficult any more. Understanding that damage changes the dynamic characteristics of a structure, it is certain structural dynamics modification is closely related to damage detection algorithm. Actually, damage in a structure can be understood as losing stiffness thus potential energy of a structure is generally reduced as damage goes to process.

Though many methods have been developed to detect damage using vibration theory, most of the methods can be divided into two groups, reference based method and experiment based method (Park, 2003). As the name says, reference based method requires a reliable model to detect damage successfully and it is known that a good reliable model is very difficult to construct. On the other hand, experiment based method does not need any reference model but requires only experimental data. But as we know, experimental work accompanies many kinds of difficulties and its applicability is very limited. 
Force balance method is one of the most well known reference based methods. In force balance method, the multiplication of reference model and measured motion vector gives useful information of damage position. In real situation, but, the force batance method can not be used frequently because there are some problems in both model reliability and coordinate mismatch between model and measurement points.

Recently improved force balance method has been suggested by the authors to overcome the problems (Park, 2003). According to the work, the force balance method can be processed with only measured frequency responses. The method utilized frequency weighting function to filter out the unwanted frequency data to ease ill-conditioning process. Though the improved force balance method does not need analytical model anymore, it needs a lot of effort to do experiments.

The prime interest in damage detection is to know damage location but the equivalent importance lies in identifying its level. Zimmerman (Kaouk and Zimmerman, 1994) tried to estimate damage extent using MRPT (Minimum Rank Perturbation Theory), and static test data are used to assess level of damage with optimization process (Sanayei and Onipede, 1991).

This work is to suggest further improved damage detection method based on force balance method. The suggesting method can relieve experimental load significantly and also damage extent can be estimated from measured data. Substructure damage detection is the key of the paper and is based on substructure modification (Maia and Silva, 1998).

\section{Background Theory (Park, 2003)}

Considering a dynamic system having $n$ degrees of freedom (dofs), the response vector $\{X\}_{n \times 1}$ can be divided into two parts, $m$ dofs measured responses $\left\{X_{m}\right\}_{m \times 1}$ and the remain unmeasured responses $\left\{X_{u}\right\}_{(n-m) \times 1}$. If the external forces acting on the unmeasured dofs are zero, the incompletely measured frequency response matrix can be written as

$$
\left[H_{m m}\right]_{m \times m}=\left(\left[D_{m m}\right]-\left[D_{m u}\right]\left[D_{u u}\right]^{-1}\left[D_{u m}\right)_{m \times m}^{-1}\right.
$$

where $[D]$ is dynamic stiffness matrix, $-\omega^{2}[M]+$ $i \omega[C]+[K]$, which is composed of mass, stiffness and damping matrix. The subscript ' $m$ ' means measured, and ' $u$ ' unmeasured.

Eq. (1) says that the incompletely measured frequency response function matrix $\left[H_{m m}(\omega)\right]$ is equal to the inverse of reduced dynamic system matrix which can be derived from an exact analytic model. When some damages occur in structure, the reduced dynamic system matrix is somewhat changed. Thus the force balance equation can be written as

$$
\begin{aligned}
& {\left[D_{m m}-\left(D_{m u} D_{u u}^{-1} D_{u m}\right)\right]\{\tilde{H}\}_{j}} \\
& =\{I\}_{j}-\left[\Delta D_{m m}-\Delta E\right]\{\widetilde{H}\}_{j}
\end{aligned}
$$

where $\{I\} ;$ is an unit vector which has the value of one only in the $j$-th position and zeros elsewhere. $\left[\Delta D_{m m}\right]$ and $[\Delta E]$ is the variation of $\left[D_{m m}\right]$ and $\left[D_{m u} D_{u t}^{-1} D_{u m}\right]$ respectively due to damage, and $\{\bar{H}\}_{j}$ is the $j^{\text {-th }}$ column of the measured frequency response of damaged structure. The left-hand side of Eq. (2) can be determined from the reduced dynamic stiffness matrix of undamaged structure and measured frequency responses from damaged structure, and both are already known. On the other hand, the right hand side of the equation contains an unknown term $\left[\Delta D_{m s t}-\Delta E\right](\tilde{H}\}_{j}$, which comes from damage and it measures the deviation from $\{I\}_{j}$. Thus the change of unit vector $\{I\}_{j}$ due to damage can be treated as an index to indicate damage locations. Introducing a vector $\{\xi\}$, the deviation of the unit vector $\{I\}_{j}$ can be defined as

$$
\{\xi\}=\left[H_{m m}\right]^{-1}\{\widetilde{H}\}_{j}-\{I\}_{j}
$$

The right-hand side of Eq. (3) can be determined from only experimental data. Thus exact theoretical model is no longer necessary.

Although the proposed method does not require any analytic model, it does not work well to identify damage through all the frequency ranges. That is because experimental model is a kind of reduced model, it contains numerical errors generated in inverse processes. Thus an additional process to examine the quality of each frequency is needed. A new vector $\{\eta\}$, which 
Table 1 Some temarks on the proposed damage detection method

\begin{tabular}{c|c|c}
\hline \hline & previous force balance method & proposed method \\
\hline $\begin{array}{c}\text { reliability } \\
\text { D.F. mismatch } \\
\text { difficulty }\end{array}$ & $\begin{array}{c}\text { dependent on model quality } \\
\text { reduction or expansion is required } \\
\text { difficult to build an accurate model }\end{array}$ & $\begin{array}{c}\text { dependent on experimental results } \\
\text { any reduction or expansion is not required } \\
\text { heavy experimental burden }\end{array}$ \\
\hline
\end{tabular}

is the summation of weighted damage detection vector $\{\xi(f)\}$ through interesting frequency ranges, is defined as equation (4)

$$
\{\eta\}=\sum_{i=1}^{q} \sum_{j=1}^{r} W\left(f_{s_{i j}}\right)\left\{\xi\left(f_{s_{i}}\right)\right\}
$$

where $\left\{\xi\left(f_{s_{\ell}}\right)\right\}$ is defined in Eq. (3), and $f_{s_{s_{l}}}$ is $j$-th frequency of $i$-th set of the selected frequency region. When computing $\{\eta\}$, if we use some frequencies where $W(f)$ has a high value, then the damage identification result becomes more reliable. For the cases of having multiple damages it is recommendable to select several frequency sets because natural frequency sensitivity differs with damage locations. It is also reasonable to avoid the frequencies where its condition number is very high. A guide to determine weighting function, $W(f)$, is introduced in the previous work (Park, 2003), and some remarks on the proposed method is listed in Table 1.

It is interesting to note that the characteristics of dynamically reduced model have a direct relationship with those of unmeasured whole model. In fact, determinant of the measured frequency response functions can be written as

$$
\operatorname{det}\left(\left[H_{m m}\right]\right)=\frac{\operatorname{det}\left(\left[D_{u u}\right]\right)}{\operatorname{det}([D])}
$$

And it is easily noticed that the solution of $\operatorname{det}\left(\left[H_{m m}\right]\right)=0$ satisfies the other form of characteristic equation $\operatorname{det}\left(\left[D_{u u}\right]\right)=0$. Specifically the frequencies at which $\operatorname{det}\left(\left[H_{m m}\right]\right)=0$ are known as transmission zeros. Since the reduced dynamic system is ill-posed at the transmission zeros, those frequencies should be avoided when computing its inverse.

\section{Damage Detection Based on Substructure Diagnosis}

Generally it is well known that a system has a large condition number at its natural frequencies and transmission zeros because the system is ill-posed at those frequencies. And these frequencies are easily found from measured frequency response functions. Actually, as will be shown in Eq. (6), this work assumes that the Frobenious norm of $\left[D_{m m}\right]$ is greater than the norm of $\left[D_{m u} D_{u u}^{-1} D_{u m}\right]$. If not, we cannot expect a reliable damage detection result as can be seen in Eq. (2). This paper, thus, implicitly assumes that

$$
\left\|\left[D_{m m}\right]\right\| \gg\left\|_{i}\left[D_{m u} D_{u u}^{-1} D_{u m}\right]\right\|
$$

Although the relationship between $\left\|\left[D_{m m}\right]\right\|$ and $\left\|\left[D_{m u} D_{u u}^{-1} D_{u m}\right]\right\|$ cannot be derived without an exact model, it is right that this inequality is not true at the frequencies which satisfy $\operatorname{det}\left(\left[D_{u u}\right]\right)=$ 0 . Judged from some numerical and experimental tests, it is expected that the proposed damage detection algorithm leads to a confident result at the frequencies where the condition number of the measured frequency response function $\left[H_{m m}\right]$ is relatively low. If the inequality relation is satisfied in a substructure domain, it will be very helpful to relieve experimental load. This section shows that the effect of $\left\|\left[D_{m u} D_{u u}^{-1} D_{u m}\right]\right\|$ in a subdomain can be ignored as like that of entire domain, thus the proposed algorithm can be applied.

Reduced substructure model can be defined as Eq. (7)

$$
\left[D_{m m}-D_{m u} D_{u w}^{-1} D_{u m}\right]_{S}^{i} \equiv[H]_{s}^{-1}
$$

where superscript ' $\mathrm{i}$ ' with subscript ' $\mathrm{S}$ ' means $i$-th substructure. Since Eq. (7) is identical to Eq. (1), the damage detection method suggested in this work can be applied to substructures too. The advantage of using substructures is that the inspection space is dramatically reduced. 
Considering a finite element structure which can simulate exact dynamics of a structure, it is obvious that the experimental reference model is directly related to the number of measurement. Therefore, at a given frequency, an experimental model can be thought as a function of the number of measurcment ' $m$ ', then it leads to Eq. (8)

$$
\left\|\left[D_{m m}(\omega)\right]_{s}\right\|_{\omega \omega_{0}}=f(m)
$$

where $\left\|\left[D_{m m}(\omega)_{s}\right]\right\|$ is the norm of dynamic stiffness of a substructure. Let us examine a symmetric dynamic system with $m+u$ dofs like Eq. (9),

$$
\left[\begin{array}{cc}
D_{t r m} & D_{m u} \\
D_{u m} & D_{u u}
\end{array}\right]=\left[\begin{array}{ccc}
D_{(m-e)(m-e)} & D_{\langle m-e) e} & D_{(m-e) u} \\
D_{e(m-e)} & D_{e e} & D_{e u} \\
D_{u(m-e)} & D_{u e} & D_{u u}
\end{array}\right]
$$

where ' $e$ ' means the number of reduced measurement points. From the definition of Frobenious norm, Eq. (10) can be derived from Eq. (9).

$$
\begin{aligned}
\left\|\left[D_{m m}\right]\right\|^{2}= & \left\|\left[D_{(m-e)(m-e)}\right]\right\|^{2}+2\left\|\left[D_{(m-e) e}\right]\right\|^{2} \\
& +\left\|\left[D_{e e}\right]\right\|^{2}
\end{aligned}
$$

Thus when the subsystem with reduced measurement points, $\left[D_{m m}\right]_{s}$, is set to $\left[D_{(m-e)(m-e)}\right]$, Eqs. (8) and (10) comes to

$$
f^{2}(m)=\left\|\left[D_{m m}\right]\right\|^{2}-2 \Delta_{1}-\Delta_{3}
$$

where $\Delta_{1}, \Delta_{3}$ is the square norm of $\left[D_{(m-e) e}\right]$ and $\left[D_{e e}\right]$, respectively. Eq. (11) means that measurement points diminishment causes norm reduction of the measured set. The coupled system $\left\|\left[D_{m u} D_{u u}^{-1} D_{u m}\right]_{s}\right\|^{\|}$also satisfies the following inequality that is

$$
\begin{aligned}
& \left\|\left[D_{m u} D_{u u}^{-1} D_{u m}\right]_{s}\right\| \leq\left\|\left[D_{m u}\right]_{s}\right\|^{2}\left\|\left[D_{u t u}^{-1}\right]_{s}\right\| \\
& =\frac{\mathcal{c}\left(\left[D_{u u}\right]_{s}\right)\left\|\left[D_{m u}\right]_{s}\right\|^{2}}{\left\|\left[D_{u u}\right]_{s}\right\|}
\end{aligned}
$$

where $c\left(\left[D_{u z}\right]_{s}\right)$ is condition number of $\left[D_{u s}\right]_{s}$. If $g_{1}(m)$ and $g_{2}(m)$ represents the norm of $\left\|\left[D_{m u}\right]_{s}\right\|$ and $\|\left[D_{u t u}\right]$ s $\|$ respectively, their relation can be summarized as Eqs. (13) and (14).

$$
\begin{gathered}
g_{1}^{2}(m)=\left\|\left[D_{m u}\right]\right\|^{2}+\Delta_{1}-\Delta_{2} \\
g_{2}^{2}=\left\|\left[D_{u u}\right]\right\|^{2}+2 \Delta_{2}+\Delta_{3}
\end{gathered}
$$

Where $\Delta_{2}$ is the square norm of $\left[D_{e t}\right]$. If the number of measurement points decrease, surely $g_{2}(m)$ will increase. Using Eqs. (13) and (14), Eq. (12) becomes to

$$
\begin{aligned}
& \left\|\left[D_{m u} D_{u u}^{-1} D_{u m}\right] s\right\| \leq \frac{c\left(\left[D_{u t}\right] s\right)}{g_{2}(m)} g_{1}^{2}(m) \\
& =\frac{c\left(\left[D_{u u}\right]_{s}\right)\left(\left\|\left[D_{m u}\right]\right\|^{2}+\Delta_{1}-\Delta_{2}\right)}{\sqrt{\left\|\left[D_{u u}\right]\right\|^{2}+2 \Delta_{2}+\Delta_{3}}}
\end{aligned}
$$

Assuming smoothly varying condition number, $c\left(\left[D_{u u}\right]_{s}\right)$, the upper bound of Eq. (15) will decrease as the number of measurement points decrease. Except several extraordinary frequencies having relatively large condition number, it is numerically verified that $\left\|\left[D_{m m}\right]_{s}\right\|$ is larger than $\left\|\left[D_{m u} D_{u u}^{-1} D_{u m}\right]_{s}\right\|$.

Consequently, it is moderate to divide the entire domain of a structure into several subdomains to relieve experimental load, and each subdomain will be examined carefully through the suggested damage detection algorithm. Also the idea to select proper frequencies (Park, 2003) can be directly applied to damage detection in subdomains.

\section{Estimation of Damage Severity}

\subsection{Sensitivity analysis and consideration of degrees of freedom mismatch}

Though the prime interest for damage detection is to identify damage location, it is also important to estimate the level of damage. Severity of damage cannot be known only with frequency response functions. Therefore some damage coefficients which link the severity with measured responses must be considered.

Ignoring damping, eigenvalue equation of a system before damage can be expressed as

$$
[K]\{\phi\}=\lambda[M]\{\phi\}
$$

where $\lambda$ is an eigenvalue and $\{\phi\}$ is its modeshape vector. If damage is engaged in a structure and assuming the damage affects on stiffness only, the above equation can be changed to

$$
\left([K]-\sum_{i=1}^{L} p_{i}[K]_{i}-\bar{\lambda}[M]\right)\{X\}=\{0\}
$$


where $L$ is the number of damage, $\tilde{\lambda}$ and $\{X\}$ mean varied eigenvalue and eigenvector caused by damage. Also the parameter, $p_{i}$, determines severity of damage and has a value in the range of 0 to 1 . If we express the damage, $\sum_{i=1}^{L} p_{i}[K]_{i}$, as $[K(\vec{p})]$ and rearranging the equation, it leads to

$$
([I]-[H(\mu(\vec{p}))][K(\vec{p})])(X\}=\{0\}
$$

where $[I]$ is identity matrix and $\mu$ means circular natural frequency of modified system. And $[H(\mu(\vec{p}))]$ is frequency response function before damage. Eq. (19) which is the determinant equation of Eq. (18) provides natural frequencies (Tsuei and Yee, 1989).

$$
\operatorname{det}([I]-[H(\mu(\vec{p}))][K(\vec{p})])=0
$$

It is also interesting to note that if both frequency response functions and damage model are known, modified system's eigenvalue can be found. If we define $\{Y\}$ as a left eigenvector of the system Eq. (18), then it satisfies another form of eigenvalue equation as

$$
\{Y\}^{T}([I]-[H(\mu(\vec{p}))][K(\vec{p})])=\{0\}^{T}
$$

Eq. (19) and (20) say that eigenvalues are a function of damage parameter $\vec{p}$. Thus it is possible to assign appropriate eigenvalues by controlling damage parameters. Natural frequency sensitivity, therefore, acts an important role in identifying damage severity. Differentiating both sides of Eq. (18) about $p$, one obtains the following equation

$$
\begin{aligned}
& \left(\frac{\partial[H(\mu(\vec{p}))]}{\partial p}[K(\vec{p})]+[H(\mu(\vec{b}))] \frac{d[K(\vec{b})]}{d p}\right)(X) \\
& =([I]-[H(\mu(\vec{p}))][K(\vec{p})]) \frac{d(X)}{d p}
\end{aligned}
$$

Further applying chain rule, and multiplying left eigenvector $\{Y\}$ to Eq. (21), and combining with Eq. (20), natural frequency sensitivity with respect to a parameter can be derived

$$
\begin{aligned}
\frac{d \mu}{d p}= & -\{Y\}^{T}[H(\mu)] \frac{d[K(\vec{p})]}{d p}\{X\} \\
& \left(\{Y\}^{T} \frac{\partial[H(\mu)]}{\partial \mu}[K(\vec{p})]\{X\}\right)^{-1}
\end{aligned}
$$

Actually Eq. (22) is not useful, because Eq. (22) requires completely measured frequency response functions to match with degrees of freedom of damaged substructure model $[K(\vec{p})]$, while this work is based on partially measured frequency response functions. If damage model dofs is $m$, Eq. (19) can be rewritten as

$$
\operatorname{det}\left(\left[I_{m m}\right]-\left[H_{m m}(\mu(\vec{p}))\right]\left[K_{d}(\vec{b})\right]\right)=0
$$

Thus if a well defined damage model $\left[K_{d}(\vec{p})\right]$ is provided, natural frequency change can be predicted using incompletely measured frequency response functions.

Still there is another problem to be solved; how can we define damage element which is suitable for measured frequency responses. It is natural to consider dynamically reduced damage model, actually it is equivalent to that of static reduction because damage is assumed only in stiffness. Thus desired damage model, $\left[K_{d}(\vec{p})\right]$, can be written as

$$
\left[K_{d}(\vec{p})\right]=\left[K_{d}\right]_{m m}-\left[K_{d}\right]_{m u}\left[K_{d}\right]_{u u}^{-1}\left[K_{d}\right]_{u m}
$$

Therefore the provided damage model has the same size of incompletely measured frequency response functions.

\subsection{Design optimization problem}

Estimation of damage extent can be understood as a design optimization problem. In this case, the objective function to be minimized is the summation of natural frequency differences between before and after damage occurrence. The optimization problem can be defined as

$$
\begin{aligned}
& \min _{p \in K^{2}} \frac{1}{2} \Lambda(\vec{p})^{\tau} \Lambda(\vec{p}) \\
& \text { subject to } \\
& 0 \leq p_{i} \leq 1
\end{aligned}
$$

where $\vec{p} \in R^{L}$ and $\Lambda \in R^{N}$. The residual function $A: R^{L} \rightarrow R^{N}$ is nonlinear in $p$, and means difference vector between target and current natural frequencies. $L$ is the number of most probable damage candidates and $N$ is the number of target natural frequency after damage.

The optimization problem needs a solution technique for nonlinear optimization problem, 
and generally iteration is indispensable. The soIution of Eq. (25) is obtained withou: difliculty by applying Gauss-Ncwion's melhod iteratively, and ustialiy the solution of each iteration is

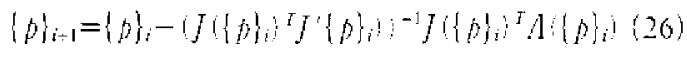

where $f$ means Jacobian, natural liequency sensitivity vector. Since the Jacobian of Eq. (26) is not always well conditioned. it is also well known fatet that Gatuss-Newton's method does nol globally convergent. Thus many modiiled Newton's methods are developed. and this nork ried to find an optimum solution by applying Levenberg-Marqualdr method (Dennis and Schnabel. 1983).

Solution or design parameter denoted $\vec{b}$ will be designed to minimize the error. The number of design parameters can be selected on the criterion that comes from damage location index. Those parameters may not indicate exact damage location, since there should be some identification error. Thus, through the procedure of severity estimation, one can nol only deduce appropriate damage level but also recheck actually damaged region.

\section{Test Examples}

\subsection{Plate structure}

A test structure is freely suspended on wires as showl in Fig. 1, and 30 points are set for mea-

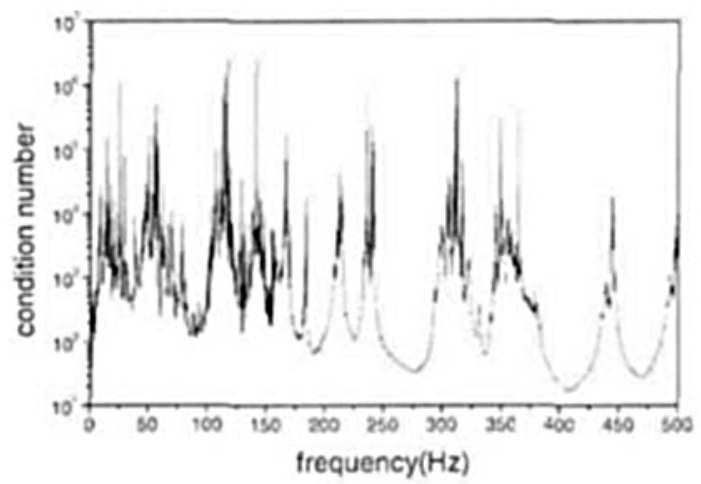

(a) sutement. The steel plate hat $1.6 \mathrm{~mm}$ thickness and saw cul with length of $35 \mathrm{~mm}$ to make a damage near moasurement points 19,20,24 and 25.

The cotal number of experiment to build an experimental model is 900 if the enire domain is considered. But loat is too many to do experiment and thus the structure is divided into wo purts. One part is composed of 20 points from poin 1 to point 20). the other part is composed of 15 points from point 16 to point 30 . So the number of measurement 10 construct substructure model

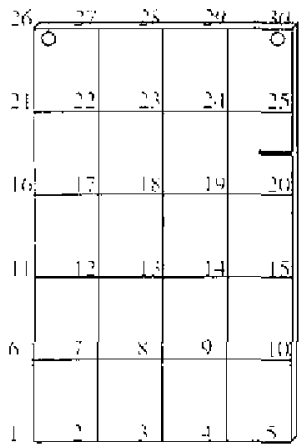

Fig. 1 Stecl plate $280 \times 400 \times 1.6 \mathrm{mml}$ with $35 \mathrm{~mm}$ length saw cul damage

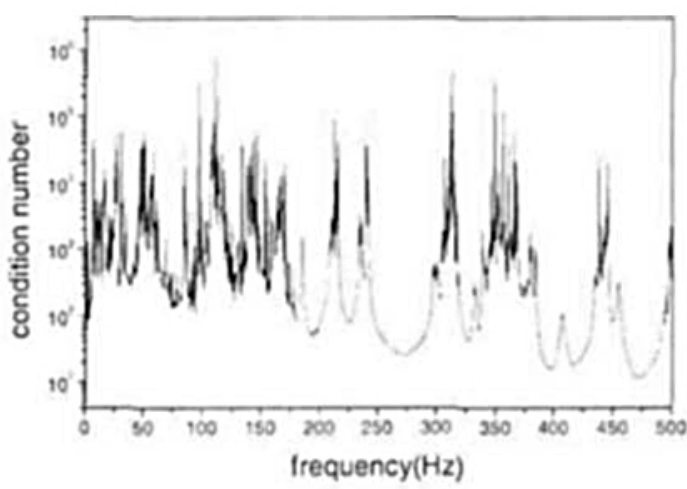

(b)

Fig. 2 Condition number of substructures. (a) Condition number of $20 \times 20$ frequency response matrix of the first substructure. (b) Condition number of $15 \times 15$ frequency fosponse matrix of the second substructure 
can be reduced to 400 and 225 respectively.

Condition number of each case is delineated in Fig. 2, and it seems that there is no abrupt difference in these two figures except near $410 \mathrm{~Hz}$. It is thought that this frequency is a transmission zero of the second substructure. The weighting functions for these two substructures are shown in Fig. 3. From these weighting functions two frequency ranges, $220 \sim 250 \mathrm{~Hz}$ and $420 \sim 450 \mathrm{~Hz}$, are selected for inspection and some frequencies with higher noise level than 0.05 or with higher condition number than 300 are discarded to avoid ill-pose. Since the actual measurement error is not known, this work assumed normalized random error using coherence function (Bendat and Piersol, (993).

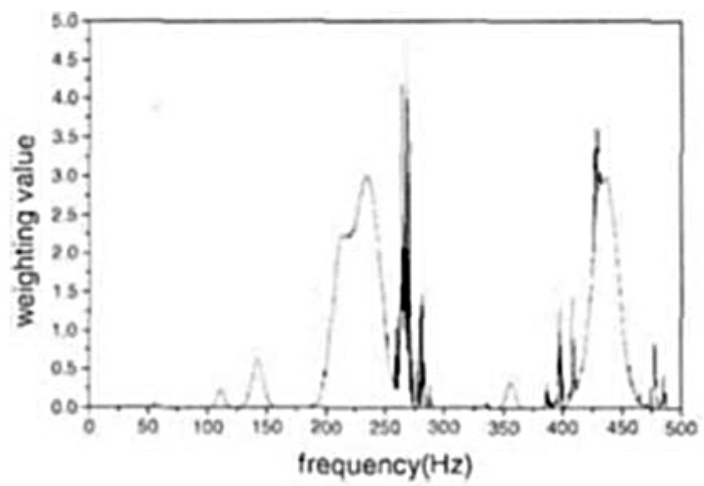

(a)
Using Eq. (4), damage location indices of the two substructures are found in Fig. 4. True damage exists in the second substructure, and it is easily found in Fig. 4(b). Though there exists no damage in the first substructure, Fig. $4(a)$ indicates that it might have damaged zones. The suspicious damage zone shown in Fig. $4(\mathrm{a})$ is resulted from the effect of the true damage in the second substructure, because the indices with high value are distributed around the true damage region.

The first step, to identify damage level, is to build a reliable analytic structure model. For improving reliability of the damage model, 4 global design parameters, Young's modulus, density, Poisson's ratio, and thickness, were chosen.

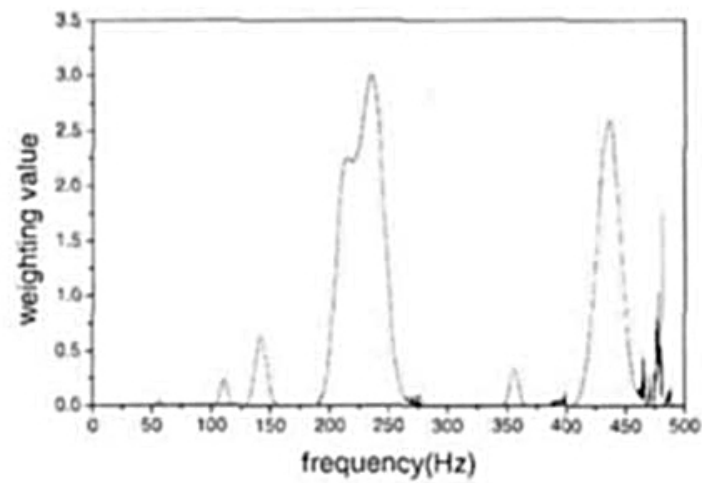

(b)

Fig. 3 Weighting function of each substructure. (a) Weighting function of the first substructure. (b) Weighting function of the second substructure

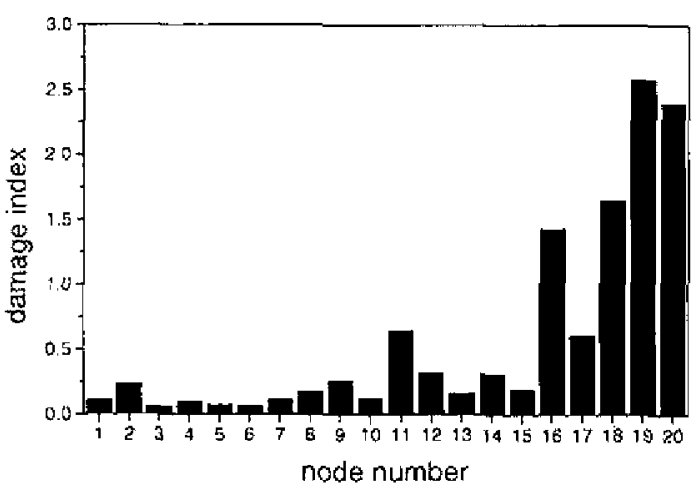

(a)

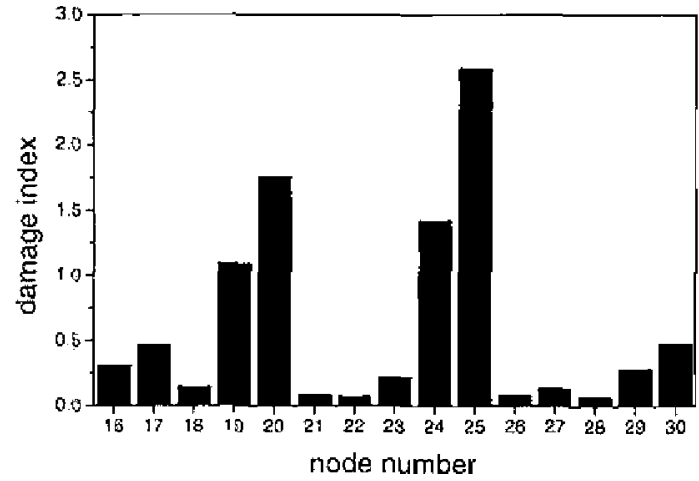

(b)

Fig. 4 Danage location indices using two sets of frequency $220-250 \mathrm{~Hz}, 420-450 \mathrm{~Hz}$ (a) Damage location index of the first substructure. (b) Damage location index of the second substructure 
Table 2 Natural frequency of a plate example

\begin{tabular}{c|c|c|c|c}
\hline \hline \multirow{2}{*}{$\begin{array}{c}\text { experimental } \\
\text { resuli }(\mathrm{Hz})\end{array}$} & \multicolumn{2}{|c|}{ before update } & \multicolumn{2}{c}{ after update } \\
\cline { 2 - 5 } & frequency $(\mathrm{Hz})$ & relative error $(\%)$ & frequency $(\mathrm{Hz})$ & relative error $(\%)$ \\
\hline 47.61 & 46.21 & 2.93 & 48.06 & 0.95 \\
55.91 & 51.96 & 7.05 & 54.04 & 3.33 \\
111.22 & 108.87 & 2.11 & 113.22 & 1.80 \\
118.90 & 110.81 & 6.80 & 115.24 & 3.07 \\
142.57 & 135.84 & 4.72 & 141.28 & 0.90 \\
166.30 & 157.43 & 5.33 & 163.73 & 1.54 \\
\hline
\end{tabular}

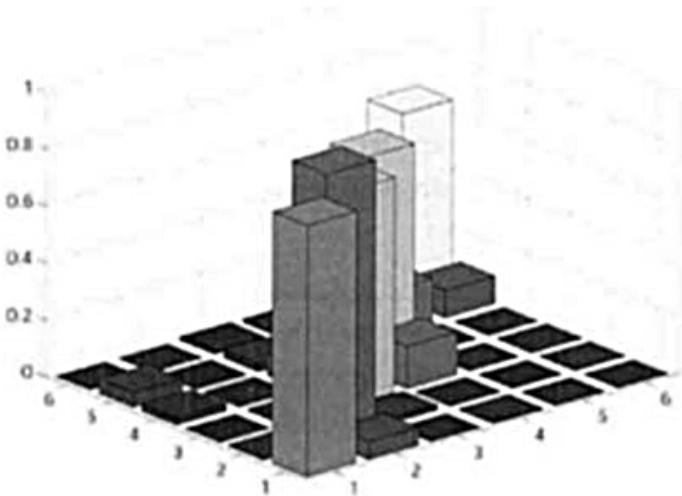

(a)

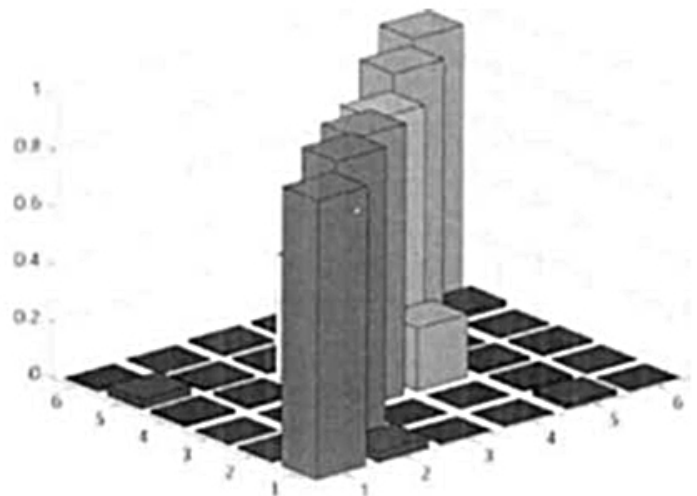

(b)

Fig. 5 MAC value of plate. (a) Before update. (b) After update

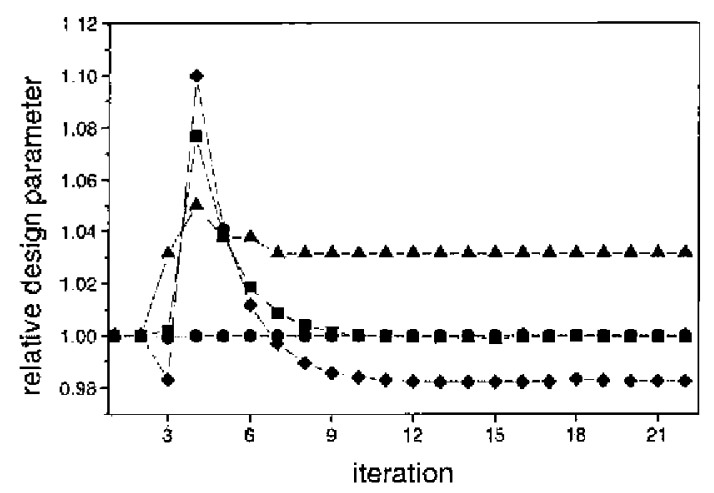

Fig. 6 Relative design parameter change ratio. Young's modulus (relative to $200 \times 10^{9} \mathrm{pa}$ ) ; $\rightarrow$ Poisson's ratio (relative to 0.33 ) ; $\rightarrow$ density (relative to $7860 \mathrm{~kg} / \mathrm{m}^{3}$ ); - thickness (relative to $0.0016 \mathrm{~m}$ )

The objective function, the error of the first six natural frequencies is minimized by adjusting these four parameters. The result is shown in Fig. 5 and Table 2. It can be seen that the updated model is reliable enough to identify damage level. Updated design parameters are also shown in Fig. 6.

Though, in this case, the damaged area is exactly detected, it is assumed that some other arex is disguised as damage. Two damage parameters, thus, are selected and tested to locate damage positions. That is the true damage area composed of points 19, 20,24 and 25 is set to the first candidate and the area, beneath the true damage area, composed of points $14,15,19$ and 20 as the second candidate. First three natural frequency error between the damaged experiment model and updated model are set as objective function. Applying above damage level identification procedure, Eq. (25), it can be said that true damage area is clearly found as shown in Fig. 7 .

\subsection{Structure with joints}

The plate structure that was tested is severed into two parts as shown in Fig. 8. The two parts 


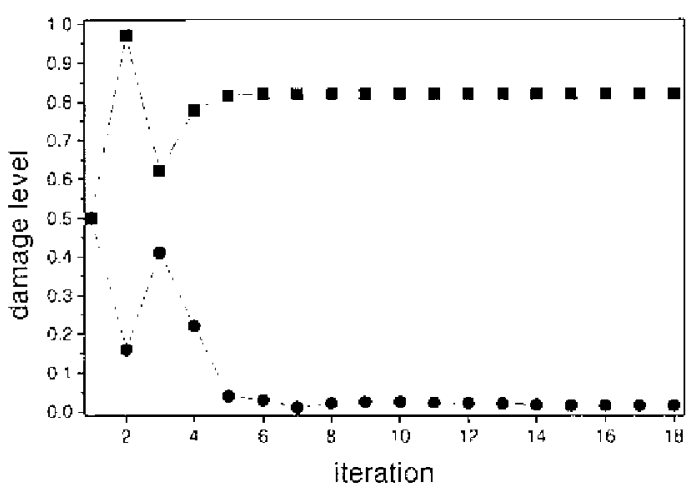

Fig. 7 Estimated plate damage level caused by saw cutting. - The first damage candidate :

The second damage candidate : Note that true damage area can be detected by considering the fact a false candidate converges to zero approximately

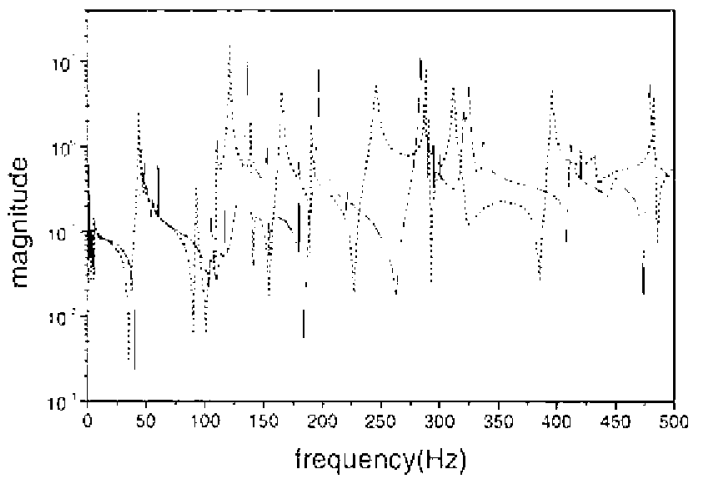

(a)

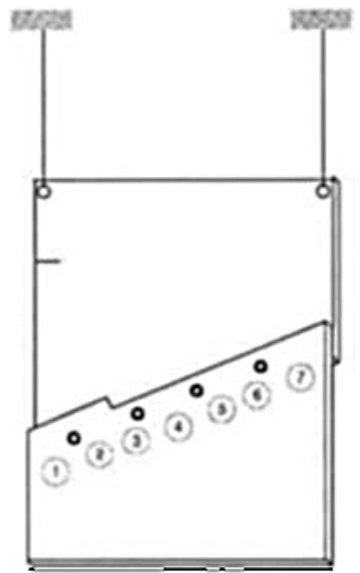

Fig. 8 Previous plate structure was severed into two, the rwo plates are coupled with 4 bolts and nuts: the number in the circle means measurement position

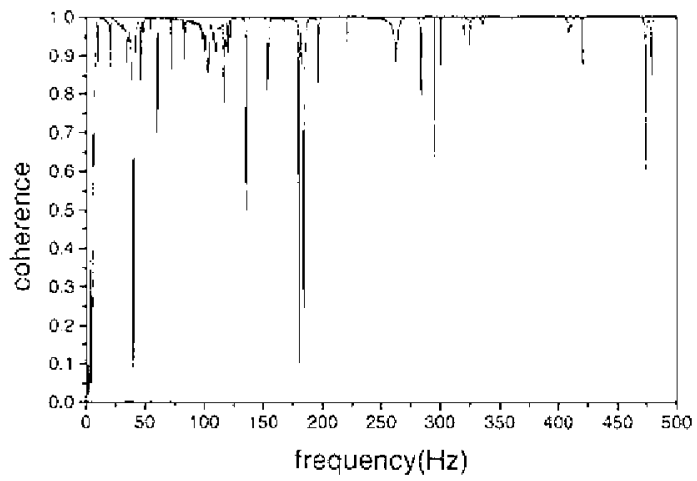

(b)

Fig. 9 Measured signals (a) A frequency response function before ( - ) and after damage (----); $H_{1,2}$ (b) Coherence function of the measured frequency response function

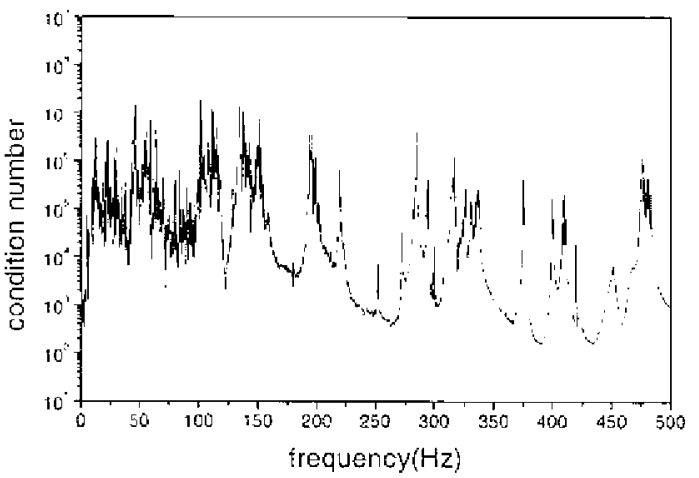

(a)

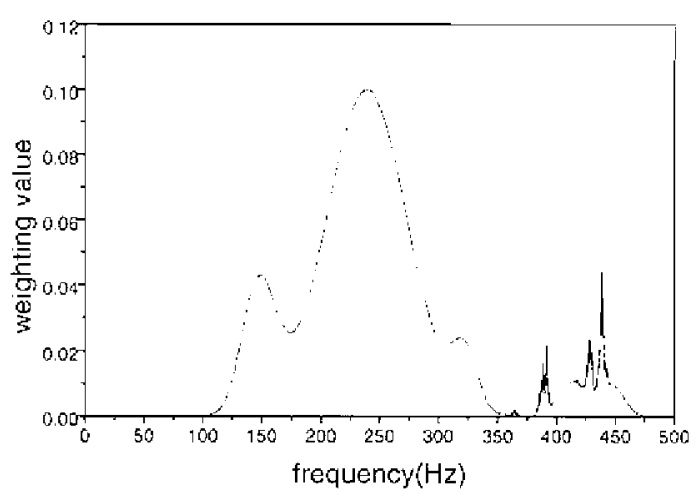

(b)

Fig. 10 Condition number and weighting function for the coupled structure. (a) Condition number of $7 \times 7$ frequency response matrix. (b) Weighting function 
are coupled with 4 bolts and nuts. The first joint from the left is released so as to simulate damage. 7 measuremen! points were selected and acceleration of each point was measured.

The total number of measurement to construct experimental model is 49 . Fig. 9 is a sample of frequency response functions and its coherence. Condition numbers of the frequency response matrix, before damage, is delineated in Fig. 10(a). The weighting function of the system is shown in Fig. 10(b). From this weighting function, frequency band $210 \sim 290 \mathrm{~Hz}$ is selected for inspection. Some lirequencies with the level of noise higher than 0.05 or with condition number higher than 800 are excluded. Based on Eq. (4), the calculated damage location index with the weighting function shows the damaged first joint cleurly as Fig. II.

Since four global design parameters were determined already at the above example, only joint stiffness is to be considered to construct a reliable model for estimation of damage severily. It is assumed that joint can be modeled as spring element thus 12 springs, one linear and two torsional springs for each joint, will be used to inprove model reliability. It is thought that the linear spring is relatively rigid comparing to torsional springs, thus 4 linear springs are set to relatively rigid. $1.0 \times 10^{10} \mathrm{~N} / \mathrm{m}$.

The objective function. the error of the first three natural frequencies, is minimized as varying the 8 torsional springs. Table 3 shows initial and moditied design parameters. Table 4 shows the updated model is reliable enough to identify damage level.

The next stcp is to estimate damage level. In this case, it is matural to consider 3 spring constants in the released joint as damage parameters. With the same procedure, the damage level can be identified as shown in Fig. 12. It is interesting to note that linear spring constant approaches $100 \%$ damage level, since a released joint is equivalent to decoupling of lateral motion.

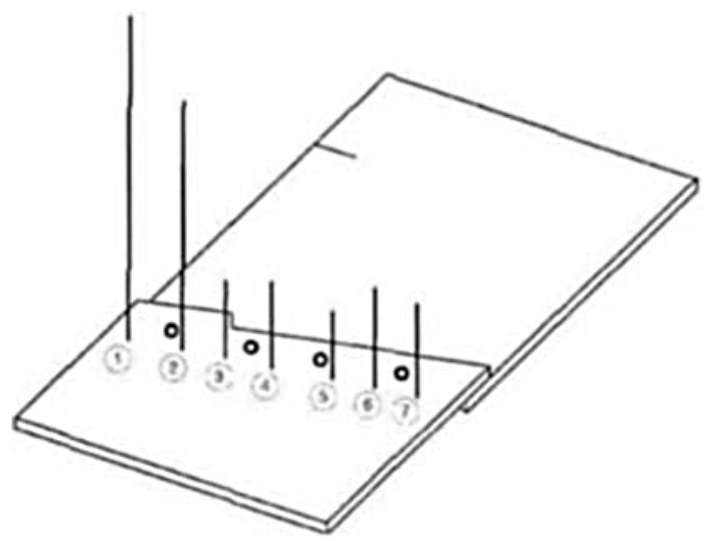

Fig. 11 Weighted damage location index: vertical bar means damage index normalized to the maximum

Table 3 Linear $(\mathrm{N} / \mathrm{m})$ and torsional $(\mathrm{Nm} / \mathrm{rad})$ spring constants

\begin{tabular}{c|ccc|ccc}
\hline \multirow{2}{*}{$\begin{array}{c}\text { parameter from } \\
\text { the left }\end{array}$} & \multicolumn{3}{|c|}{ initial } & \multicolumn{3}{c}{ modified } \\
\cline { 2 - 7 } & $\mathrm{kz}$ & $\mathrm{krx}$ & $\mathrm{kry}$ & $\mathrm{kz}$ & $\mathrm{krx}$ & $\mathrm{kry}$ \\
\hline $\mathrm{P1}$ & $1.000 \mathrm{E} 10$ & $1.000 \mathrm{E} 3$ & $1.000 \mathrm{E} 3$ & $1.000 \mathrm{E} 10$ & $1.0068 \mathrm{E} 5$ & $9.6626 \mathrm{E} 4$ \\
$\mathrm{P} 2$ & $1.000 \mathrm{E} 10$ & $1.000 \mathrm{E} 3$ & $1.000 \mathrm{E} 3$ & $1.000 \mathrm{E} 10$ & $1.0148 \mathrm{E} 5$ & $9.8757 \mathrm{E} 4$ \\
$\mathrm{P} 3$ & $1.000 \mathrm{E} 10$ & $1.000 \mathrm{E} 3$ & $1.000 \mathrm{E} 3$ & $1.000 \mathrm{E} 10$ & $9.8973 \mathrm{E} 4$ & $9.8690 \mathrm{E} 4$ \\
$\mathrm{P} 4$ & $1.000 \mathrm{E} 10$ & $1.000 \mathrm{E} 3$ & $1.000 \mathrm{E} 3$ & $1.000 \mathrm{E} 10$ & $9.6703 \mathrm{E} 4$ & $9.7193 \mathrm{E} 4$ \\
\hline
\end{tabular}

Table 4 Natural frequency of a plate with joints

\begin{tabular}{|c|c|c|c|c|}
\hline \multirow{2}{*}{$\begin{array}{l}\text { experimental } \\
\text { resul }(\mathrm{Hz})\end{array}$} & \multicolumn{2}{|c|}{ before update } & \multicolumn{2}{|c|}{ after update } \\
\hline & liequency $(\mathrm{Hz})$ & relative error $(\%)$ & frequency $(\mathrm{Hz})$ & relative error $(\%)$ \\
\hline 40.3 & 49.5 & 0.9 & 46.8 & 1.1 \\
\hline 53.4 & 56.2 & 5.2 & 54.0 & 1.1 \\
\hline 104.3 & 109.8 & 5.3 & 104.5 & 0.02 \\
\hline
\end{tabular}




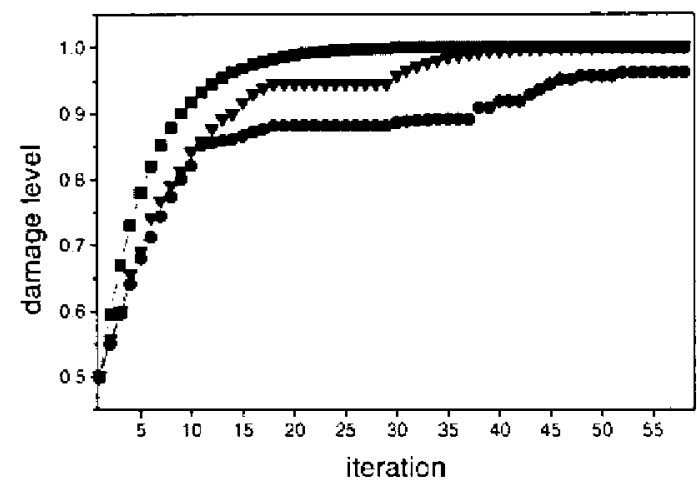

Fig. 12 Estimated joint damage level caused by loosed joint.,$-- \mathrm{kz} ;-\bullet, \mathrm{krx} ; \rightarrow-, \mathrm{kry}$

\section{Conclusions}

In this paper, substructure damage identification method and estimation of damage level is suggested. The previously proposed algorithm with measured frequency response functions is totally dependent upon experimental data, thus it takes much time and effort to acquire experiment data. To relieve experimental load, substructure damage detection is suggested and this process helps to reduce experimental load effectively. Identification of damage level is also important and an optimization problem is defined to estimate damage level. The estimation process needs only measured frequency response function and statically reduced stiffness matrix.

Two test examples are introduced to verify suggested method, and the results show that suggested method is confident.

\section{References}

Bendat, J. S. and Piersol, A. G., 1993, "Engi- neering Application of Correlation and Spectral Anlysis," John Wiley \& Sons Inc.

Choi, Y. J. and Lee, U., 2003, "An Accelerated Inverse Perturbation Method for Structural Damage Identification," KSME Int. Journal, 17(5), pp. $637 \sim 646$.

Dennis, J. E. and Schnabel, R. B., 1983, "Numerical Methods for Unconstrained Optimization and Nonlinear Equations," Prentice-Hall Inc..

Doebling, S. W., Hemez, F. M., Peterson, L. D. and Fahrat, C., 1997, "Improved Damage Location Accuracy Using Strain Energy-Based Mode Selection Criteria," AIAA journal, 35(3), pp. 693 699.

Kaouk, M. and Zimmerman, D.C., 1994, "Structural Damage Assessment Using a Generalized Minimum Rank Perturbation Theory," AIAA Journal, 32(4), pp. 836 842.

Maia, N. M. M. and Silva, J. M. M., 1998, "Theoretical and Experimental Modal Analysis," Research Studies Press LTD.

Marwala, T, and Heyns, S., 1998, "MultipleCriterion Method for Determining Structural Damage," ALAA Journal, 36 (8), pp. 1494 - 1501.

Park, N. G. and Park, Y. S., 2003, "Damage Detection Method Using Spatially Incomplete Frequency Response Functions," Mechanical Systems and Signal Processing, 17(3), pp. 519 -532.

Sanayei, M. and Onipede, O., 1991, "Damage Assessment of Structures Using Static Test Data," AIAA Journal, 29(7), pp. 1174 1179.

Tsuei, Y.G. and Yee Eric, K. L., 1989, "A Method for Modifying Dynamic Properties of Undamped Mechanical Systems," J. Dynamic Systems, Measurement and Control, ASME, 111, pp. $403-408$. 